\title{
DISEASE MODELING WITHIN REFUGEE CAMPS: A MULTI-AGENT SYSTEMS APPROACH
}

\author{
Andrew Crooks \\ Ates Hailegiorgis \\ Department of Computational Social Science \& Center for Social Complexity \\ George Mason University \\ Fairfax, VA 22030, USA
}

\begin{abstract}
The displacement of people in times of crisis represents a challenge for humanitarian assistance and disaster relief and stakeholder agencies. Major challenges include providing adequate security and medical facilities to displaced people. Within this paper, we develop a spatially explicit multi-agent system model that explores the spread of cholera in the Dadaab refugee camps, Kenya. A common characteristic of these camps is poor sanitation and housing conditions which contribute to frequent outbreaks of cholera. We model the spread of cholera by explicitly representing the interaction between humans (host) and their environment, and the spread of the epidemic. The results from the model show that the spread of cholera grows radially from contaminated water sources and can have an impact on service provision. Agents' social behavior and movements contribute to the spread of cholera to other camps where water sources were relatively safe.
\end{abstract}

\section{INTRODUCTION}

The displacement of people in times of crisis represents a challenge for humanitarian assistance and disaster relief (HADR) and stakeholder agencies. People either become displaced by man-made and natural disasters such as drought, flooding, ethnic conflict, and political violence. The United Nations High Commissioner for Refugees (UNHCR 2010) Global Trend report indicated that about 44 million people were displaced worldwide in 2010 with the majority being in developing countries. This displacement of people challenges humanitarian agencies who attempt to provide basic services (such as food, water and healthcare) at camps or other locations to ease the suffering of these displaced people. The displacement of people is especially acute in Africa, where the most common causes of death has been diarrheal diseases, measles, acute respiratory infections and malaria (Toole and Waldman 1997). Many of which are preventative in the developed world.

Cholera is an acute problem in Africa especially when related to refugee camps (e.g. Siddique 1994; Swerdlow et al. 1997) as such places often suffer from poor sanitation and low per capita availability of water (Cronin et al. 2008). Within this paper we focus one such camp complex. That of the Dadaab refugee camp complex which is located near the Kenya-Somalia border in the North Eastern Province of Kenya as shown in Figure 1. This camp complex hosts nearly half a million of refugees (UNHCR 2012) with a high influx of new refugees coming from Somalia due to drought, famine, and violence. The camps have poor sanitation and housing conditions with frequent incidences of cholera outbreaks (see UNHCR 2011) among with other diseases (e.g. measles). In the remainder of the paper we discuss prior work with respect to cholera, refugee camps and disease modeling more generally (Section 2) before introducing our multi-agent system (MAS) model in Section 3. Section 4 presents some preliminary results from our work while in Section 5 we provide a summary and identifies future research avenues. 


\section{Crooks and Hailegiorgis}

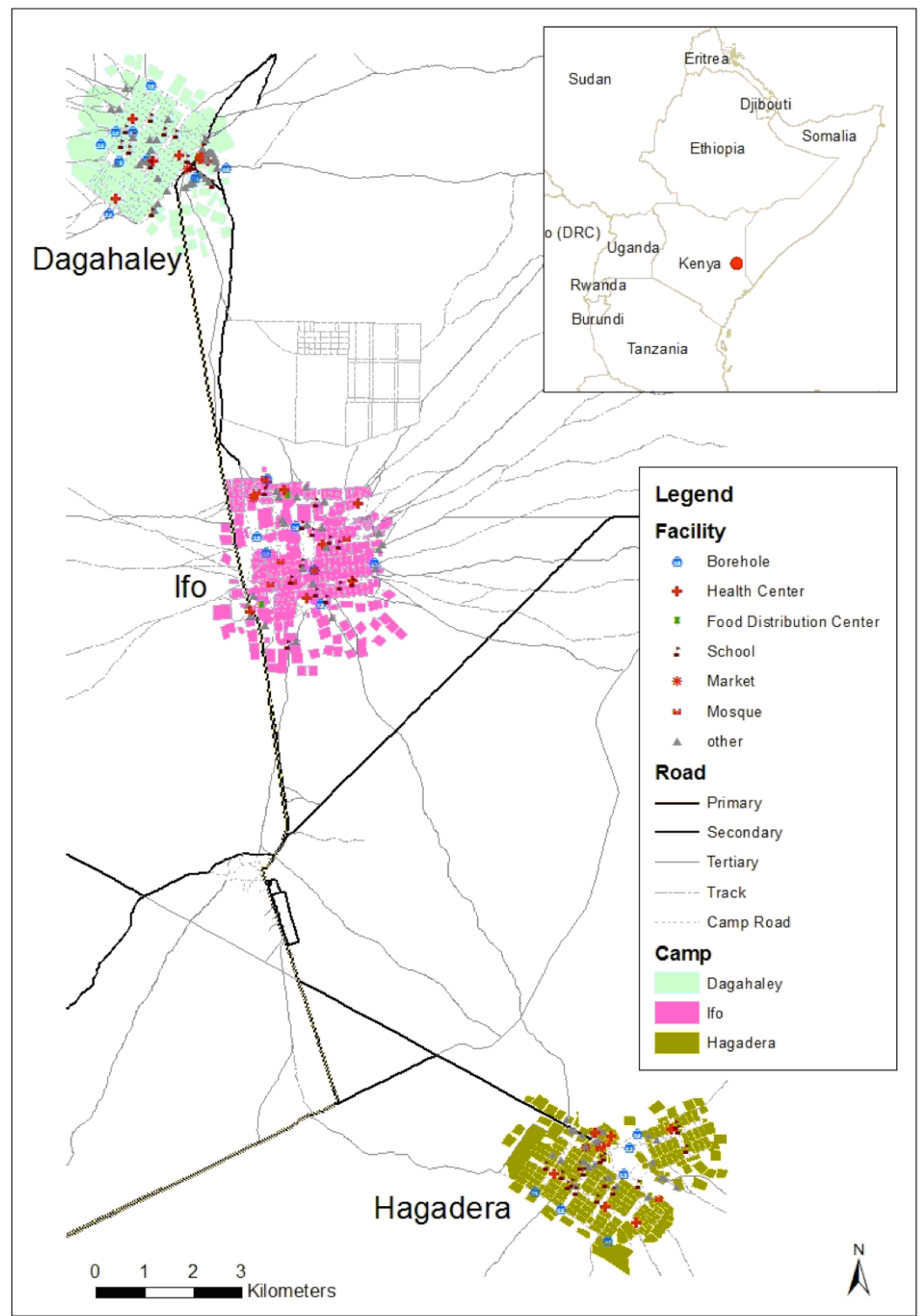

Figure 1: Location of the Dadaab refugee camps.

\section{BACKGROUND}

Cholera is an intestinal disease caused by the bacterium Vibrio cholerae, which colonizes the human intestine (Bertuzzo et al. 2010) which is characterized by diarrhea and severe dehydration. The main transmission mechanism for cholera is by drinking water or eating food contaminated by $V$. cholerae which enters the system via feces (stools) from infected people. Even though cholera itself is both preventable and treatable via the treatment of raw sewage or by providing clean drinking water, using oral cholera vaccines or once infected using rehydration therapy. It remains a health hazard in many developing countries where such care or prevention is not possible. It is extremely difficult to obtain the actual numbers of deaths per year due to under or no reporting, but it is estimated that cholera kills between 100,000150,000 people per year (Longini et al. 2007). Once cholera arrives into a new region, either carried by an infected person or by contaminated water and food, three different scenarios might be seen. The first is 


\section{Crooks and Hailegiorgis}

that there is no outbreak; the second is an outbreak might occur which is then followed by few waves of outbreaks; while in the third scenario, an outbreak might occur which is then followed by subsequent outbreaks persistent seasonal pattern (Codeço 2001).

While cholera has a long tradition of being mapped and modeled using spatial analysis techniques (e.g. Oseia et al. 2010; Lobitz et al. 2000), modeling the propagation and the spread of the disease on the human population has had a much briefer history. But such models are needed as they play a crucial role in the study of infectious diseases and as a tool for exploring what might happen and experimenting with different control strategies (Fisman 2009). This relates to the notion that while spatial analysis techniques provides powerful insights they are limited with respect to modeling/capturing spatial interaction of people, and one might argue that even spatial autoregressive measures used as surrogates for such interaction as in the work of Oseia et al. (2010) are no substitute for actual agent mixings as seen within MAS models (Crooks and Heppenstall 2012). With respect to cholera models, there are several existing models, many of which are aggregate mathematical models using differential equations (e.g. Codeço 2001). More recently a number of spatially explicit mathematical models have been developed (e.g. Longini et al. 2007; Bertuzzo et al. 2010; Tuite et al. 2011). While such styles of models have proved useful they have also been criticized by Epstein (2009) for being ill-suited to model complex human systems. This relates to the notion that such models do not incorporate direct contact between individuals or their environment, too often they assume uniform mixing assumptions which is not the case as people interact with each other in many different ways (Crooks and Heppenstall 2012). Moreover, they treat people as aggregate individuals missing the heterogeneity of the human population and key individual based behaviors.

MAS models have been applied to a number of epidemiological problems ranging from the spread of the swine flu (Epstein 2009) to that of mumps (Simoes 2012). With respect to MAS models and cholera, to the authors' knowledge, this is one of the first applications. The other application that authors are aware of is that of the work Augustijn-Beckers et al. (2011) who extends the empirical spatial analysis work of Oseia et al. (2010) to model the spread of cholera in the city Kumasi in south central Ghana. However, our model differs in two aspects compared to this model. First we do not use a SIR model but rather a SEIR model which we go into more details about in Section 3. Secondly we are focusing on refugee camps rather than a small city where water becomes contaminated via a refuse site. Moreover, with respect to refugee camps there has been little work carried out with using MAS models.

\section{METHODOLOGY}

The purpose of the model is to explore the spread of cholera, which is caused by the interaction of human (host) with their environment in the in the Dadaab refugee camp complex. Within this section we present our agents (Section 3.1) before describing the environment upon which the agents operate (Section 3.2) and the implementation of the SEIR model (Section 3.3). A overview of the model dashboard can be seen at http://bit.ly/XGpqm6 while the full overview, design concepts, and details (ODD) protocol can be found at http://css.gmu.edu/Cholera/.

\subsection{Agents}

The main agent in the model is the refugee agent who represents an individual refugee who lives in the camps. A refugee agent has family and fixed home location. Agents of the same family cooperate and share resources. Agents are instantiated with different attributes that contribute to their heterogeneity. Agents differ in their personal characteristics (e.g. age, sex and education level), social ties (e.g. number of family members and friends), their body immunity type (symptomatic and asymptomatic), and goals and priorities. Behaviorally, agents are mobile and purpose-oriented. They determine a specific activity (goal) at a given time (which is represented in minutes), depending on their priorities, and move towards it to fulfill their satisfaction.

At each time step, each agent makes decision to stay where they are or move to their goal based on their priorities (e.g. needing food or water, to attend school). Agent movement from home to goal is de- 


\section{Crooks and Hailegiorgis}

termined by the time and their success at the goal as shown in Figure 2. Each agent moves towards his goal by selecting the shortest path based on an underlying road network. Since an agent is be constrained by time, the success of the agent to reach its goal is not guaranteed. If an agent is successful and reaches its goal, the agent might return to home if he accomplishes his activity or stays for a while until he is able to accomplish his activity before he returns to his home (case 1). For example, an agent may have to wait in line to get food aid. If the agent is too late to accomplish his goal, the agent will return home before reaching his goal (case 2). In some instants, an agent might consider choosing another goal before going back to their home (case 3). In the current version of the model, this will only happen when the agent fails to collect enough water from their nearest water point (e.g. the water point has run out of water) and are therefore forced to search for other water points to achieve its goal.

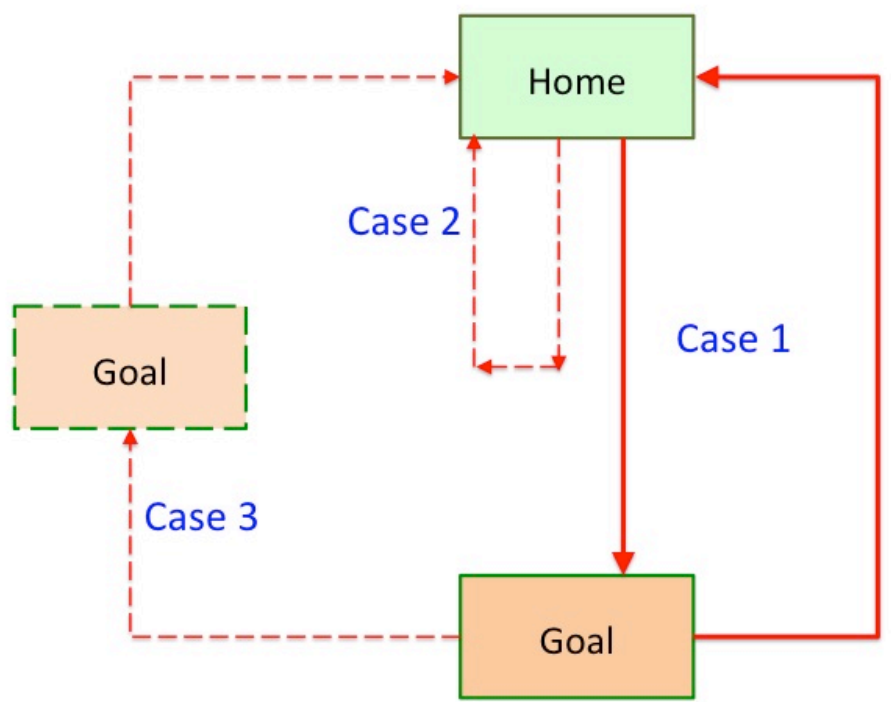

Figure 2: Agent's movement options between his home and his goal.

The dynamics of the model are driven by the interaction of agents with their environment. Agents of the same family share resources (e.g. water). Agents interact with other agents who are their relatives or friends. Agents have a fixed number of relatives, which is given to them at the initialization of the model; however, they select friends randomly at runtime.

\subsection{The Spatial Environment}

The other component of the model is the environment, which is a representation of the Dadaab refugee camp complex. It has a spatial extent of 13.5 kilometers by 25 kilometers with a spatial resolution of 90 meters by 90 meters. The spatial resolution is equivalent with an average distance that human can travel in a minute (humans can travels about 5 kilometers per hour on average, which is about 90 meters per minute). The spatial extent covers all of the three camps sites (Dagahaley, Ifo and Hagadera) located around the town of Dadaab (as shown in Figure 1). The environment encompasses field units (i.e. cells), camp boundaries, houses (e.g. tents), facilities, infrastructure, and elevation. All the spatial data was collected from publicly available sources. For example, information pertaining to camp boundaries, houses, facilities, and infrastructure were collected from UNHCR (2012). The field unit is the main unit of the environment in which all processes of the model take place. A field unit may hold up to three houses but can only hold a single facility (e.g. a borehole or a school).

Within our model we use rain as a proxy for climatic events as previous research has shown that there are strong correlations between seasons and outbreaks of cholera (see Reiner et al. 2012). Secondly water is one of the main methods that cholera is transmitted (Codeço 2001) which we discuss further in Section 
3.3. In this model, we use rainfall both as source of water for the refugee as well as a carrier of pollutants (disposal and feces). We utilize a digital elevation model (DEM) to model rainfall runoff. We apply simple hydrologic model that only considers the elevation gradient to model surface runoff. Rainfall flows downhill according to the elevation gradient. A prototype of which can be found at http://bit.ly/15gaorB. As the water flows from uphill to downhill, it carries pollutants (e.g. V. cholerae). The concentration of pollutants in the water depends on the amount of pollutant per volume of water in the field unit (parcel).

The dynamics of surface runoff is designed as follows. When there is rain, all cells get equal amount of rainfall. At each time step, each cell will check if it has water to flow to its Moore neighbors. If the cell has water and the neighboring cell is a sink (i.e. at a lower elevation), it will give the water until it fills the sink depending on the elevation and water gradient. If the volume of water is less than the sink, all the water will flow to the sink. However, if the volume of water is greater than the sink, the water will flow until the two cells reach to equal level. As rainfall flows from cell to cell, the concentration of pollutant (in this case V. cholerae) in the water varies accordingly. As this is a simple hydrological model we do not consider evaporation or infiltration mechanism within it, which we consider beyond the scope of the current study.

\subsection{Cholera SEIR Model}

A cholera epidemic is mainly caused by $V$. cholerae bacteria and the bacteria can survive in aquatic reservoirs or the host's intestine for some time. Nelson et al. (2009) described the dynamic nature of cholera by explicitly representing the interaction between host (human) and the environment (aquatic reserve), and the progress of the epidemic using a Susceptible - Infected - Recovered (SIR) model (a common approach in many epidemiological studies (e.g. Simoes 2012; Augustijn-Beckers et al. 2011). We extend their representation to Susceptible - Exposed - Infected - Recovered (SEIR) model to better capture the time between ingestion of contaminated water and showing the symptom as shown in Figure 3.

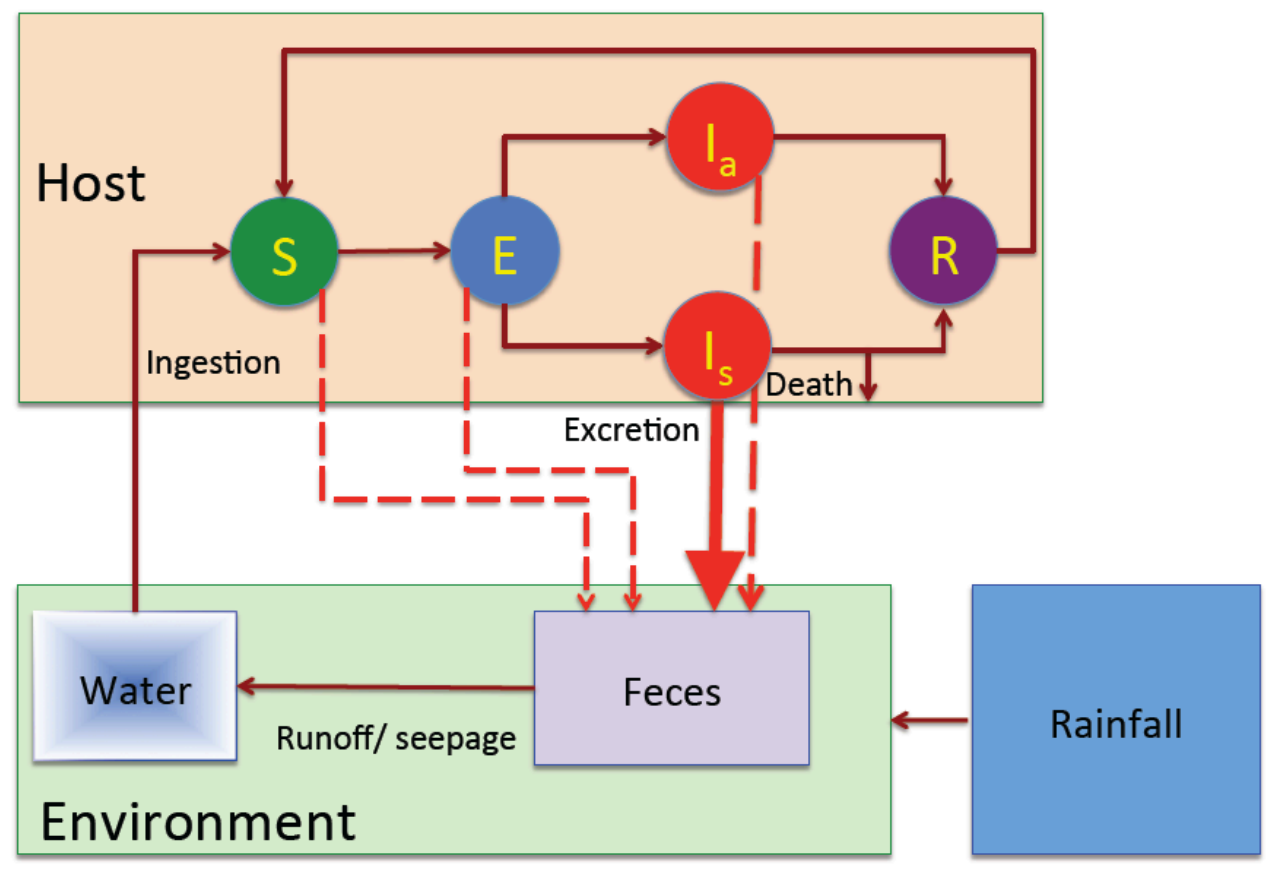

Figure 3: Cholera transmission through the interaction of host and the environment. The progress of cholera transmission is represented as SEIR model. $\mathrm{S}$ = susceptible, $\mathrm{E}=$ Exposed, Ia= Infected (asymptomatic), Is $=$ Infected (symptomatic). 


\section{Crooks and Hailegiorgis}

All the refugee agents are considered as susceptible hosts. The infectious dose of $V$. cholerae in humans varies greatly depending on the bacterial strain and the host. In many cases, a bacterial cell concentration of $103 / \mathrm{ml}$ of water is necessary to infect the host (Nelson et al. 2009). We assume that a susceptible agent who ingests contaminated water with a bacterial cell concentration of $103 / \mathrm{ml}$ of water or above will be become exposed to the cholera disease. We distinguished two types of infectious agents: symptomatic and asymptomatic. A symptomatic agent shows the symptom between 8 to 17 hours after ingestion (Nelson et al. 2009 ). Infants show the symptom more quickly than adults. However, if the agent is asymptomatic, the agent will pass the infection phase to the recovery stage without showing any symptoms. In reality most infected people have no or mild symptoms but are still carriers of the disease for up to two weeks (Longini et al. 2007), while others who show symptoms can die from dehydration within hours. Agents who show symptoms of cholera, seek medical attention over everything else.

\section{RESULTS}

Based on the current status of the model, we present results from two experiments that are focusing on the spread of cholera caused by two potential sources of water contamination within the refugee camp complex. In the first scenario (Section 4.1) we contaminate one borehole within the Ifo camp with cholera which could be associated with say a leak from a ventilated improved pit. While in the second scenario (Section 4.2) we introduce rain into the model and allow agents to drink both surface water and water from boreholes to explore how rainfall impacts on the spread of cholera. Both of these scenarios act as a way of testing the inner validity of the our model. Once the model is validated we will then focus on various intervention strategies, such as restricting peoples movement around the different camps to see if the spread of the disease can be mitigated.

\subsection{Scenario 1 - Contamination of Fixed Point}

In this scenario, we consider the possibility of contamination of a drinking source through seepage. We explore the spatial distribution of cholera by infecting one of borehole in the center of the Ifo camp exogenously and run the model for five days. Within this scenario agents can only drink from boreholes. Figure 4 shows a summary from this scenario with the average results and variations between model runs. In the early stages of the model runs, the cholera infections are focused around the Ifo camp, specifically residents who live within close proximity to the contaminated borehole. However, as the model allows agents to move between camps one notices outbreaks spreading radially outwards and occurring in the other camps with a temporal lag. However, the spread of cholera fails to affect agents who are living far from the contaminated water source, except for few agents who visited their relatives or friends in the contaminated area and shared resources (i.e. contaminated water) as discussed in Section 3. However, the outbreaks with the other camps are not as severe, as one would expect. As time progresses within this scenario cyclic incidents of cholera outbreaks are observed but with fewer cases when compared to the initial outbreak. This reflects the day and night patterns of activity within the model (e.g. sleeping at night and therefore not drinking water). The number of agents who passed to the recovered stage progressively increased during this scenario and the probability getting infected again for agents after receiving treatment was minimal due to their acquired immunity.

Although agents were able to get treatment easily, there were cases of fatalities in periods where infection level is high (e.g. at the beginning of the outbreak). However, the number of deaths is not linear when compared to the number of infected agents. Two factors may contribute for this variation. First, health facilities have limited capacity, in the sense they can only treat a specific number of patients per hour. Secondly, agents might of delayed their visit health facilities due to their priority of activities (i.e. queuing at the health facilities) and eventually loses their body resistance before receiving treatment. The spatial pattern of cholera was very localized around the contaminated water source as shown in the movie at http://bit.ly/ZnWEHk. 


\section{Crooks and Hailegiorgis}

\subsection{Scenario 2 - Contamination Through Runoff}

In this scenario, we assume that the $V$. cholerae bacteria is already in the environment (e.g. carried by a person or in contaminated food) and that agents can chose to take water from either boreholes or from surface water after it rains. We introduce rain every 30 days and run the model for a 1 year time period. As can be seen from Figure 5, waves of cholera outbreaks occur as discussed in Section 2. The number of exposed and infected individuals peak after it rains, however, the larger peaks in the number of infected individuals occur during months 7 and 9 . This relates to the fact as more people become infected, more of the $V$. cholerae bacteria enters the system via them using open field latrines for their ablutions thus causing the probability of becoming exposed to increase. Overtime the infection rates decrease as many individuals are no longer susceptible to the cholera as they have already been exposed. Unlike in Section 4.1, the numbers of infected individuals is roughly the same within the 3 camps as shown in Figure 6 . If new agents where added to the model, a greater number of infections might have been observed towards the end of the year.

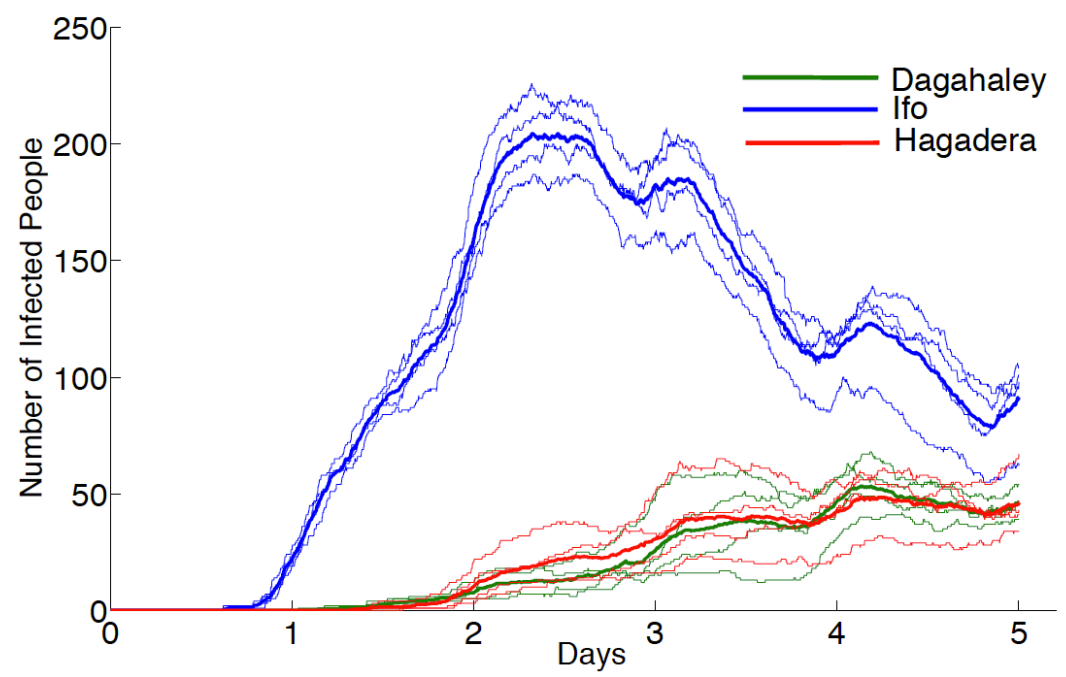

Figure 4: Distributions of cholera infections between the three camps that comprise of the Dadaab complex: Ifo (blue), Dagaheley (green) and Hagadera (red).

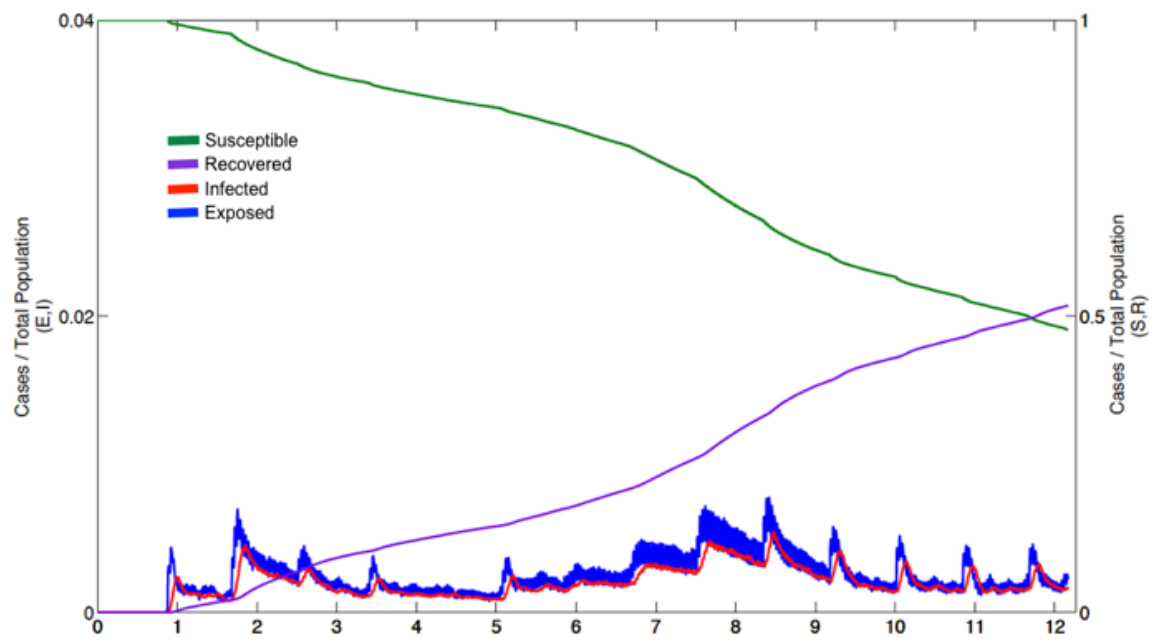

Figure 5: Cholera dynamics over a year where rain in introduced every 30 days. 


\section{Crooks and Hailegiorgis}

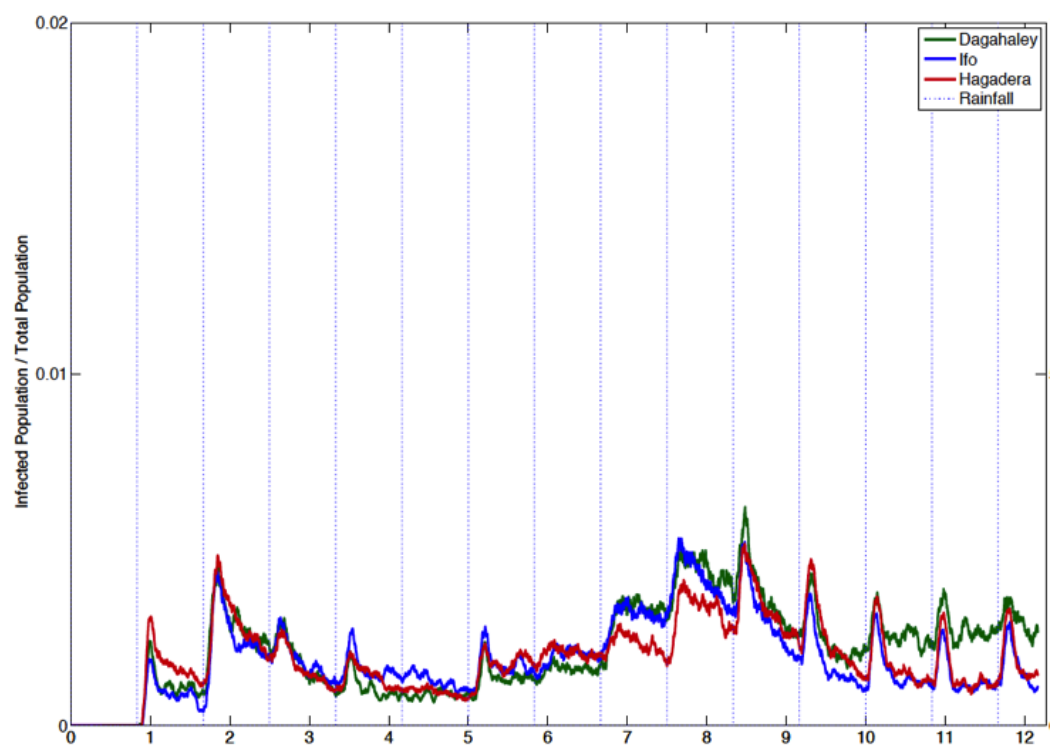

Figure 6: Distributions of cholera infections between the three camps that comprise of the Dadaab complex: Ifo (blue), Dagaheley (green) and Hagadera (red).

\section{SUMMARY}

Although the model is still under development, it yields striking results on the spread of cholera in refugee camps. It clearly demonstrates how cholera can spread through the complex interaction between humans and their environment and how agents who are exposed to contaminated water are very susceptible to cholera infection. The agents are also some of the causes of the epidemic when they use open field latrines as was the case of Scenario 2 (Section 4.2). Moreover, the model also showed that cholera cases were identified in a place where water sources were relatively safe as was the case of Scenario 1 (Section 4.1). This was due to agents travelling throughout the camps to visit their friends and relatives. Such individuals visiting areas where the cholera epidemic had occurred would then become infected and then carry it back to their homes. This signifies that although a humanitarian agency might able to contain the spread of cholera at the source by treating the contaminated water, it could be challenging to eradicate the spread of cholera as the bacteria could traveled far either by an infected person or through runoff.

This modeling effort also highlights the potential of MAS modeling to explore the spread of cholera in humanitarian contexts. MAS modeling is helpful in exploring the intensity and spatial extent of the spread of cholera. It also showed the dynamics of the spread of cholera that emerges from the complex interaction of humans and their environment. As individuals make their own decision in their daily routine and interact with other individuals and/or the environment, they act as a victim or a cause for cholera epidemic. With respect to ongoing work, we are attempting to validate our model with actual events within the Dadaab refugee camp complex (e.g. utilizing actual rainfall data and matching it to cholera outbreaks). Furthermore we are improving the dilution mechanism of $V$. cholerae bacteria in surface runoff along with optimizing the codebase to improve the runtime of model. Once these have been done the model can then be potentially used to aid understanding of the spread of cholera and to help develop control strategies for when cholera breaks out.

\section{ACKNOWLEDGMENTS}

This work was supported by the Center for Social Complexity at George Mason University and by the Office of Naval Research (ONR) under a Multi- disciplinary University Research Initiative (MURI) grant 


\section{Crooks and Hailegiorgis}

no. N00014-08-1-0921. Thanks to Stephen Prior, MD, for assistance with medical data on cholera and related epidemiology of infectious diseases. The opinions, findings, and conclusions or recommendations expressed in this work are those of the authors and do not necessarily reflect the views of the sponsors.

\section{REFERENCES}

Augustijn-Beckers, E., J. Useya, R. Zurita-Milla, and F. Osei. 2011. "Simulation of Cholera Diffusion to Compare Transmission Mechanisms." In Proceedings of the 11th International Conference on Geocomputation, 39-42. University College London, London, England, pp..

Bertuzzo, E., R. Casagrandi, M. Gatto, I. Rodriguez-Iturbe, and A. Rinaldo. (2010), “On Spatially Explicit Models of Cholera Epidemics." Journal of the Royal Society Interface 7: 321-333.

CARE. 2012. "Horn of Africa Food Crisis." http://www.care.org/emergency/Horn-of-Africa-foodpoverty-crisis-Dadaab-2011/index.asp [Accessed on July 1, 2012].

Codeço, C. T. 2001. "Endemic and Epidemic Dynamics of Cholera: The Role of the Aquatic Reservoir." BMC Infectious Diseases 1: Available at http://www.biomedcentral.com/1471-2334/1/1.

Cronin, A. A., D. Shrestha, N. Cornier, F. Abdalla, N. Ezard, and C. Aramburu. 2008. "A Review of Water and Sanitation Provision in Refugee Camps in Association with Selected Health and Nutrition Indicators - The Need for Integrated Service Provision." Journal of Water and Health 6:1-13.

Crooks, A.T. and A. Heppenstall. 2012. "Introduction to Agent-Based Modelling." In Agent-based Models of Geographical Systems, Edited by Heppenstall, A., A. T. Crooks, L. M. See, and M. Batty, 85108, New York: Springer.

Epstein, J. M. 2009. "Modelling to Contain Pandemics." Nature, 460: 687.

Fisman, D. 2009. "Modelling an Influenza Pandemic: A Guide for the Perplexed." Canadian Medical Association Journal 181:171-173.

Lobitz, B., L. Beck, B. Huq, B. Wood, G. Fuchs, S. M. Faruque, and R. Colwell. 2000. "Climate and Infectious Diseases: Use of Remote Sensing for Detection of Vibrio Cholerae by Indirect Measurement." Proceedings of the National Academic Sciences 97:1438-1443.

Longini, I. M., A. Nizam, M. Ali, M. Yunus, and N. Shenvi. 2007. "Controlling Endemic Cholera with Oral Vaccines." PLoS Med 4: e336.

Nelson, E. J., J. B. Harris, J. G. Morris, S. B. Calderwood, and A. Camilli. 2009. "Cholera Transmission: The Host, Pathogen and Bacteriophage Dynamic.” Nature Reviews Microbiology 7:693-702.

Oseia, F. B., A. A. Dukera, E. W. Augustijnb, and A. Steinb. 2010. "Spatial Dependency of Cholera Prevalence on Potential Cholera Reservoirs in an Urban Area, Kumasi, Ghana." International Journal of Applied Earth Observation and Geoinformation 12:331-339.

Reiner, R. C., A. A. Kinga, M. Emch, M. Yunusd, A. S. G. Faruque, and M. Pascuala. 2012. "Highly Localized Sensitivity to Climate Forcing Drives Endemic Cholera in a Megacity." Proceedings of the National Academy of Sciences 109:2033-2036.

Siddique, A. K. 1994. "Cholera Epidemic Among Rwandan Refugees: Experience of ICDDR, B in Goma, Zaire." Glimpse 16: 3-4.

Simoes, J. 2012. "An Agent-Based/Network Approach to Spatial Epidemics." In Agent-based Models of Geographical Systems, Edited by Heppenstall, A., A. T. Crooks, L. M. See, and M. Batty, 591-610, New York: Springer.

Swerdlow, D. L., G. Malenga, G. Begkoyian, D. Nyangulu, M. J. Toole, R. J. Waldman, D. N. Puhr, and R. V. Tauxe. 1997. "Epidemic Cholera Among Refugees in Malawi, Africa: Treatment and Transmission." Epidemiology and Infection 118:207-214.

Toole, M. J. and R. J. Waldman. 1997. "The Public Health Aspects of Complex Emergencies and Refugee Situations." Annual Review of Public Health 18:283-312.

UNHCR. 2010. "Global Trends 2010." United Nations High Commissioner for Refugees. Geneva, Switzerland, http://www.unhcr.org/4dfa11499.html [Accessed on July 1, 2012]. 
UNHCR. 2011. "Health And Nutrition Sector Update Dadaab Refugee Camps 15th to 21st October 2011." Epidemiological Week 42 Update No. 14, Nairobi, Kenya.

UNHCR. 2012. "Dadaab - World's Biggest Refugee Camp 20 Years Old." http://www.unhcr.org/4f439dbb9.html [Accessed on July 1, 2012].

\section{AUTHOR BIOGRAPHIES}

ANDREW CROOKS is an Assistant Professor in the Department of Computational Social Science at George Mason University. His research interests relate to exploring, understanding and the communication of the natural and socio-economic environments using geographical information systems (GIS), spatial analysis, Web 2.0 technologies, social network analysis (SNA), and ABM methodologies. He has coedited a book entitled "Agent-based models of Geographical Systems" which brings together a comprehensive set of papers on the background, theory, technical issues and applications of ABM within geographical systems. Dr Crooks is on the editorial board of Future Internet, and regularly reviews journal articles relating to GIS and $\mathrm{ABM}$ from leading international journals. His email address is acrooks2@gmu.edu and his web page is http://gisagents.org.

ATES HAILEGIORGIS is a PhD Student in the Department of Computational Social Science at George Mason University. His research interests are in complex human-environment systems, land use-land cover change, spatially explicit agent-based models and GIS. Currently, Ates is working on a research project that focuses on developing agent-based models of socio-ecological dynamic and conflict in East Africa. He received an MS in Geo-information Science from Wageningen University, the Netherlands and a BS in Forestry from Debub University, Wondo Genet College of Forestry, Ethiopia. Prior to starting his PhD, Ates worked in the United Nations Office for Coordination of Humanitarian Affairs (UNOCHA) and as GIS analyst in International Livestock Research Institute (ILRI). His email address is ahailegi@gmu.edu. 